\title{
Current Role of Multiparametric MRI and MRI Targeted Biopsies for Prostate Cancer Diagnosis in Germany: A Nationwide Survey
}

\author{
Matthias Saar ${ }^{\mathrm{a}} \quad$ Johannes Linxweiler ${ }^{\mathrm{a}} \quad$ Angelika Borkowetz $^{\mathrm{b}}$ Sebastian Fussek $^{\mathrm{c}}$ \\ Katerina Urbanovad Laura Bellut ${ }^{\mathrm{e}}$ Glen Kristiansen ${ }^{f}$ Bernd Wullich $^{\mathrm{e}}$ \\ on behalf of the German Prostate Cancer Consortium (DPKK) \\ aDepartment of Urology and Pediatric Urology, Saarland University, Homburg/Saar, Germany; \\ ${ }^{b}$ Department of Urology, Faculty of Medicine and University Hospital Carl Gustav Carus, Technische Universität \\ Dresden, Dresden, Germany; 'Department of Urology, University Hospital Greifswald, Greifswald, Germany;

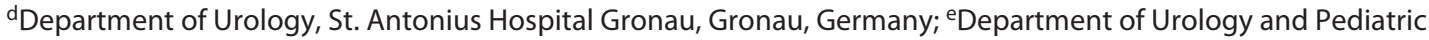 \\ Urology, Universitätsklinikum Erlangen, Friedrich-Alexander-Universität Erlangen-Nürnberg, Erlangen, Germany; \\ fInstitute of Pathology, University of Bonn Medical School, Bonn, Germany
}

\section{Keywords}

Germany - Internet survey · MRI targeted prostate biopsy ·

Multiparametric MRI · Prostate cancer · Trends

\begin{abstract}
Introduction: Multiparametric MRI (mpMRI) and MRI targeted biopsies (MRtb) are a new standard in prostate cancer (PCa) screening and diagnosis. Guidelines already include this approach for patients at risk. We aimed to gather information from German urologists about their knowledge, routine use, and attitude toward mpMRI and consecutive biopsy methods. Materials and Methods: An anonymous online questionnaire was sent via Survey Monkey to the members of the German Society of Urology (DGU). Statistical analyses were performed using SPSS version 25.0. Results: 496 members with a median age of 48.6 years $( \pm 11.7)$ participated in the survey. The majority rated $\mathrm{mpMRI}$ of the prostate as a very useful diagnostic tool (72.7\%). MRtb of the prostate was considered as very advantageous (71.5\%). MpMRI was used by $95.9 \%$, and $83.2 \%$ also recommended MRtb predomi-
\end{abstract}

\begin{tabular}{ll}
\hline karger@karger.com & $\begin{array}{l}\text { C) } 2020 \text { The Author(s) } \\
\text { Published by S. Karger AG, Basel }\end{array}$ \\
www.karger.com/uin & This is an Open Access article licensed under the Creative Commons \\
Karger & $\begin{array}{l}\text { Attribution-NonCommercial-4.0 International License (CC BY-NC) } \\
\text { (http://www.karger.com/Services/OpenAccessLicense), applicable to } \\
\text { the online version of the article only. Usage and distribution for com- } \\
\text { mercial purposes requires written permission. }\end{array}$
\end{tabular}

nantly in clinical institutions. For targeted biopsy, MRI-ultrasound fusion biopsy was clearly favored (75.8\%). MpMRI was mostly used in patients with previously negative biopsy (90.9\%) and in patients under active surveillance (60.9\%). Arguments against the use of prostate mpMRI are costs (84.9\%) and/or lack of sufficient radiological infrastructure (17.4\%). Conclusion: Our data illustrate the meanwhile high acceptance and clinical use of the prostate mpMRI and MRtb in Germany.

C 2020 The Author(s)
Published by S. Karger AG, Basel

\section{Introduction}

While there is an intense debate about the overdetection and overtreatment of low-risk prostate cancer (PCa) and the need for a more sensitive detection of clinically significant PCa, multiparametric MRI (mpMRI) is a min-

Glen Kristiansen and Bernd Wullich contributed equally.
Matthias Saar

Department of Urology and Pediatric Urology

Medical Faculty, Saarland University

Kirrberger Strasse 100, Geb. 6

DE-66421 Homburg/Saar (Germany)

matthias.saar@uks.eu 
Table 1. Characteristics of the participants

\begin{tabular}{|c|c|}
\hline Variables & $\begin{array}{l}\text { Total, } \\
n=496\end{array}$ \\
\hline Mean age (SD), years & $48.56(11.66)$ \\
\hline \multicolumn{2}{|l|}{ Gender, \% } \\
\hline Male & 82.5 \\
\hline Female & 17.5 \\
\hline \multicolumn{2}{|l|}{ Region, \% } \\
\hline Eastern Germany & 13.40 \\
\hline Western Germany & 86.60 \\
\hline \multicolumn{2}{|l|}{ Rural/urban setting, \% } \\
\hline$<20,000$ citizens & 12.02 \\
\hline $20,000-100,000$ citizens & 30.60 \\
\hline$>100,000$ citizens & 54.38 \\
\hline \multicolumn{2}{|l|}{ Practice type, $\%$} \\
\hline University & 12.15 \\
\hline Academic & 20.85 \\
\hline Communal hospital & 12.96 \\
\hline Medical practice & 54.04 \\
\hline \multicolumn{2}{|l|}{ Fully trained urologist, \% } \\
\hline Yes & 90.78 \\
\hline No & 9.22 \\
\hline \multicolumn{2}{|l|}{ Years in practice, $\%$} \\
\hline$<10$ years & 24.95 \\
\hline $10-20$ years & 28.83 \\
\hline$>20$ years & 46.22 \\
\hline \multicolumn{2}{|c|}{ Trained in PCA diagnosis and treatment, \% } \\
\hline Yes & 98.37 \\
\hline No & 1.63 \\
\hline \multicolumn{2}{|l|}{ Trained in prostate biopsy, $\%$} \\
\hline Yes & 95.10 \\
\hline No & 4.90 \\
\hline \multicolumn{2}{|c|}{ Average number of prostate biopsies per patient, $\%$} \\
\hline $1-5$ & 0.41 \\
\hline 6 & 1.45 \\
\hline $6-9$ & 1.24 \\
\hline $10-12$ & 72.20 \\
\hline $13-20$ & 19.92 \\
\hline$>20$ & 4.77 \\
\hline \multicolumn{2}{|c|}{ Average number of prostate biopsies monthly $(n=477)$} \\
\hline$<5, n(\%)$ & $160(33.54)$ \\
\hline $5-10, n(\%)$ & $187(39.20)$ \\
\hline $11-15, n(\%)$ & $49(10.27)$ \\
\hline$>15, n(\%)$ & $81(16.99)$ \\
\hline Do not use prostate MRI & $16(3.52)$ \\
\hline
\end{tabular}

imally invasive tool to identify and risk stratify patients prior to prostate biopsy [1]. Recent advances in mpMRI for prostate imaging along with the evolution of MR targeted biopsy techniques have resulted in a paradigm shift in PCa evaluation. In favor to identify more clinically significant (mostly defined as Gleason score $\geq 7 \mathrm{a}$ ) PCa cases while avoiding detection of low-risk disease, mpMRI is a very popular and widely used option.
MRI targeted biopsy (MRtb) has a proven utility in several clinical scenarios, such as in patients with prior negative transrectal ultrasound (TRUS)-guided prostate biopsy and in the monitoring of patients under active surveillance (AS) $[2,3]$. Especially lesions in areas that are mostly undersampled with TRUS biopsy (e.g., midline, anterior, and distal apical) can be thoroughly assessed by mpMRI and MRtb. Finally, the PRECISION study also forwarded MRtb as the preferable option for diagnostic workup in patients with suspicion of $\mathrm{PCa}$, who had no previous prostate biopsy [4], and this may further change clinical practice guidelines in the near future.

To the best of our knowledge, there is only very little information about the common practice for MRI diagnostics in PCa and the use of MRtb in Germany. Therefore, we assessed the current knowledge, professional attitude, and practice patterns concerning mpMRI and MRtb for PCa diagnosis among urologists in Germany.

\section{Patients and Methods}

Survey

After reviewing the literature, a 30-item questionnaire was designed to collect demographic data and information on urologists' opinion regarding the use of $\mathrm{mpMRI}$ and MRtb to detect PCa. The questionnaire contained open questions, multiple choice questions, and certain questions that allowed respondents to "select all that apply." Information was obtained on the respondents' age, gender, practice region, urban area, practice type, level of training, years in practice, number of prostate biopsies performed, and use of prostate mpMRI and MRtb in daily practice.

Study Design

A link to the survey together with a personal invitation from the German Prostate Cancer Consortium (DPKK) was sent through email to all members of the German Society of Urology (DGU). With over 6,500 members, this society is one of the largest medical societies in Germany with a heterogeneous member population of clinicians and urologic practitioners in communal and academic hospitals or in ambulant offices. Approximately 4,900 members received the email invitation. The responses were collected in an SPSS spreadsheet in an anonymous fashion.

\section{Statistics}

Statistical analyses were performed using SPSS version 25.0 (IBM, Mount Kisco, NY, USA). Univariate logistic regression analysis was performed to identify factors associated with the use of mpMRI for PCa diagnosis ("Do you use mpMRI for prostate cancer diagnosis?"), the evaluation of the usefulness of MRI targeted biopsy ("How beneficial is MRI targeted biopsy for prostate cancer detection?"), and the recommendation of MRI targeted biopsy for PCa diagnosis ("Would you recommend the use of MRI targeted biopsy for prostate cancer detection?"). Respondent's age, gender, practice region, urban area, practice type, level of training, experience in urology practice, experience in prostate biopsies, and 
Fig. 1. Distribution of participants throughout Germany.

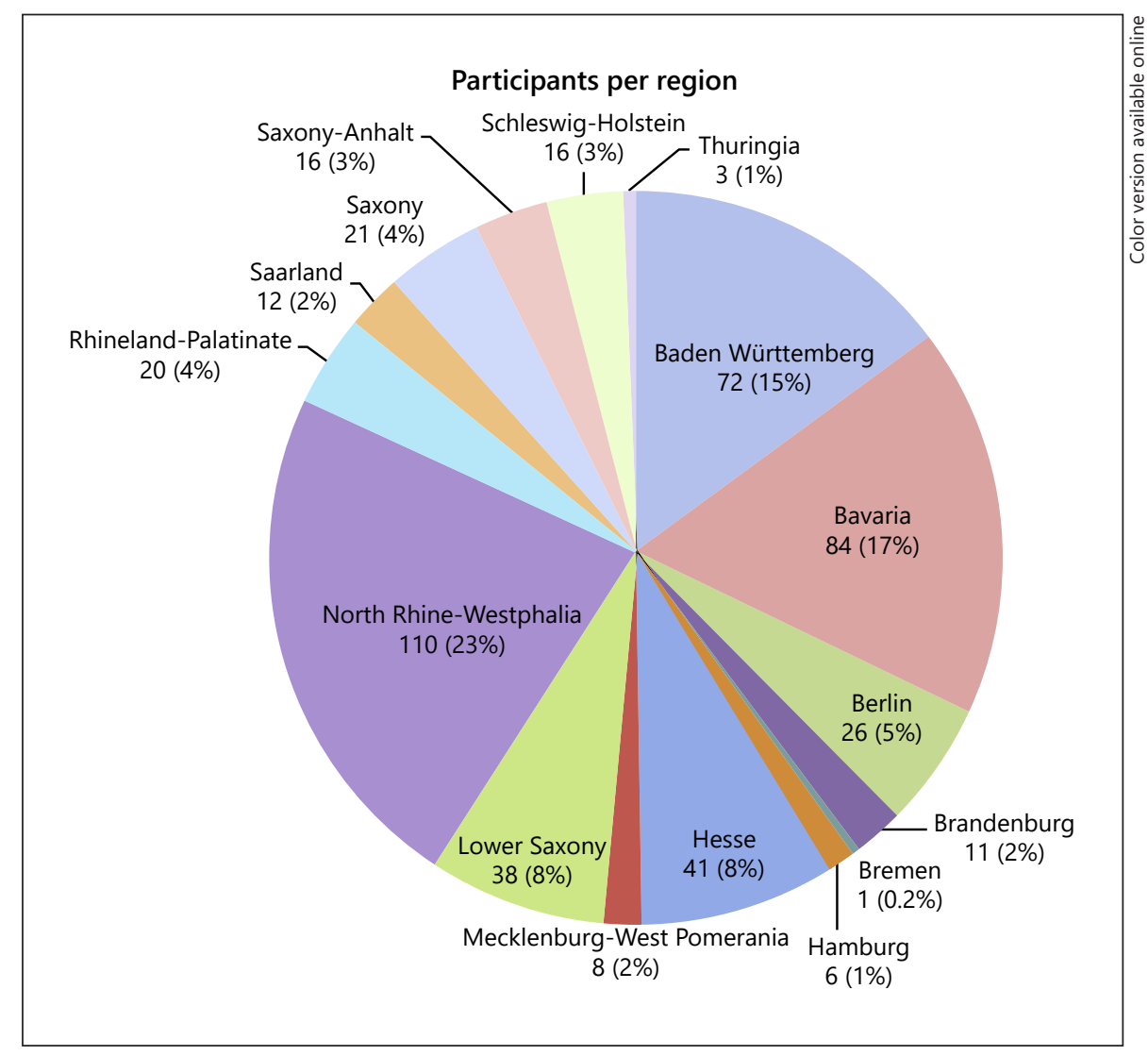

number of prostate biopsies per patient were used in regression analysis. For significant associations identified in the univariate regression analyses, all factors were identified that changed the calculated odds ratio $>10 \%$, and these were included in the respective multivariate model. Statistical significance was defined as $p<0.05$.

\section{Results}

A total of 496 responses (response rate 496/4,900; $10.1 \%)$ were received. Characteristics of the participants are indicated in Table 1. Their distribution throughout Germany is shown in Figure 1. The mean age of the participants was $48.6( \pm 11.7)$ years, $82.5 \%$ were male, and $17.5 \%$ female. $90.8 \%$ were trained urologists with an overall distribution in favor of practicing in a medical practice (54.0\%). Selected answers from the participants to the questions of the survey are shown in Figure 2. Table 2 gives an overview on the participants' answers to all survey questions. The majority of participants rated mpMRI of the prostate as very or even extremely beneficial for the evaluation of $\mathrm{PCa}(72.7 \%)$. Consequently, an MRtb of the prostate was usually rated as very or extremely advanta- geous $(71.5 \%)$. An mpMRI of the prostate was used by $95.9 \%$ of the participants, and $83.2 \%$ also recommended MRtb. Concerning the technique for MRtb, softwarebased MRI-ultrasound fusion biopsy was clearly favored $(75.8 \%)$ over a cognitive fusion biopsy $(25.9 \%)$ and "in bore" MRI biopsy (4.5\%; Table 2).

In most cases, MRtb was used in patients with previously negative prostate biopsy $(90.4 \%)$ and in patients with PCa under AS (54.6\%). 38.5\% of participants would also use MRtb in biopsy-naïve patients. In this context, German urologists stated a long waiting period for a prostate mpMRI of $2-4$ weeks $(48.1 \%)$ or even $>4$ weeks (25.5\%; Table 3). Arguments against MRtb are the costs $(77.7 \%)$ or a lack of technical infrastructure $(18.1 \%$; Table 2). Consequently, most urologists did not recommend MRtb for every patient but rather in particular patients such as those after a previous negative biopsy (Table 2). Furthermore, systematic biopsies in addition to lesiontargeted biopsies were not considered as negligible since most participants agreed in their judgment that MRtb does not eliminate the need for systematic biopsies (91\%).

On multivariate logistic regression analysis, practice in a clinic (OR $1.852[1.221 ; 2.808] ; p=0.004$ ) and a regular 
Table 2. Participants' responses to survey questions

How do you rate the use of mpMRI for prostate cancer diagnosis $(n=468)$

Very - extremely beneficial

Disadvantage - moderately beneficial

$340(72.65 \%)$

$128(27.35 \%)$

Do you use mpMRI for prostate cancer diagnosis $(n=467)$

Yes

$448(95.93 \%)$

No

$19(4.07 \%)$

Which system is used at your practice/institution to report the suspicion of prostate cancer on prostate mpMRI?a $(n=462)$

Likert

PI-RADS v1

Pi-RADS v2

None of these

Maximal number of lesions indicated in a prostate MRI $(n=457)$

$<3$

3

$>3$

How many biopsies taken per lesion? $(n=466)$

$1-2$

3-4

$\geq 5$

$5(1.08 \%)$
$74(16.02 \%)$
$390(84.42 \%)$
$20(4.33 \%)$
$67(14.66 \%)$
$197(43.11 \%)$
$193(42.23 \%)$

$210(45.06 \%)$

$232(49.79 \%)$

$24(5.15 \%)$

How beneficial is MRI targeted biopsy for prostate cancer detection? $(n=466)$

Extremely - very useful

$333(71.46 \%)$

Not - moderately useful

$133(28.54 \%)$

Would you recommend the use of MRI targeted biopsy for prostate cancer detection? $(n=465)$

Yes

$387(83.23 \%)$

$78(16.77 \%)$

Is MRI targeted biopsy better than purely systemic biopsy to detect significant cancer? $(n=468)$

Yes

$266(56.84 \%)$

No

$34(7.26 \%)$

Sometimes

Do not know

$167(35.68 \%)$

$1(0.22 \%)$

Patient selection for MRI ${ }^{\mathrm{a}}(n=496)$

Patients with no prior biopsy

$201(40.52 \%)$

Patients with prior negative biopsy

$451(90.93 \%)$

$302(60.89 \%)$

$78(15.73 \%)$

Staging before radical prostatectomy

$258(84.87 \%)$

$53(17.43 \%)$

$20(6.58 \%)$

$39(12.83 \%)$

Lack of expertise

It's no use

$142(25.97 \%)$

$21(4.50 \%)$

$353(75.75 \%)$

"In bore" MRI biopsy

MRI-ultrasound fusion biopsy

$29(6.22 \%)$

Patient selection for MR targeted biopsy ${ }^{\mathrm{a}}(n=467)$

Patients with no prior biopsy

Patients with prior negative biopsy

$180(38.54 \%)$

$422(90.36 \%)$

$255(54.60 \%)$

Patients on active surveillance

$102(21.84 \%)$

Patients prior to focal therapy

$34(7.28 \%)$

Reasons for not using MR targeted biopsy ${ }^{\mathrm{a}}(n=282)$

Cost

Lack of radiology infrastructure

$219(77.66 \%)$

$51(18.09 \%)$

Lack of expertise

$30(10.64 \%)$

It's no use

$34(12.06 \%)$ 
Table 2 (continued)

Believe in sufficient data for MR targeted biopsy use $(n=459)$

Standard for all patients

$85(18.52 \%)$

For particular patients such as prior negative biopsy

$362(78.87 \%)$

Should not be performed

$12(2.61 \%)$

Believe MR targeted biopsy eliminates the need for systematic biopsies? $(n=465)$

$38(8.17 \%)$

Yes

$423(90.97 \%)$

o not know

$4(0.86 \%)$

Believe ANNAcTRUS with targeted biopsy of suspicious lesions is a meaningful supplement to TRUS

systemic biopsies? $(n=463)$

Yes

$53(11.45 \%)$

$115(24.83 \%)$

For particular patients

$210(45.36 \%)$

No

$85(18.36 \%)$

Believe elastography is a meaningful supplement to TRUS ultrasound for the detection of suspicious

Yes

For particular patients

$78(16.85 \%)$

No

Do not know

$61(13.17 \%)$

${ }^{a}$ Multiple answers were possible.

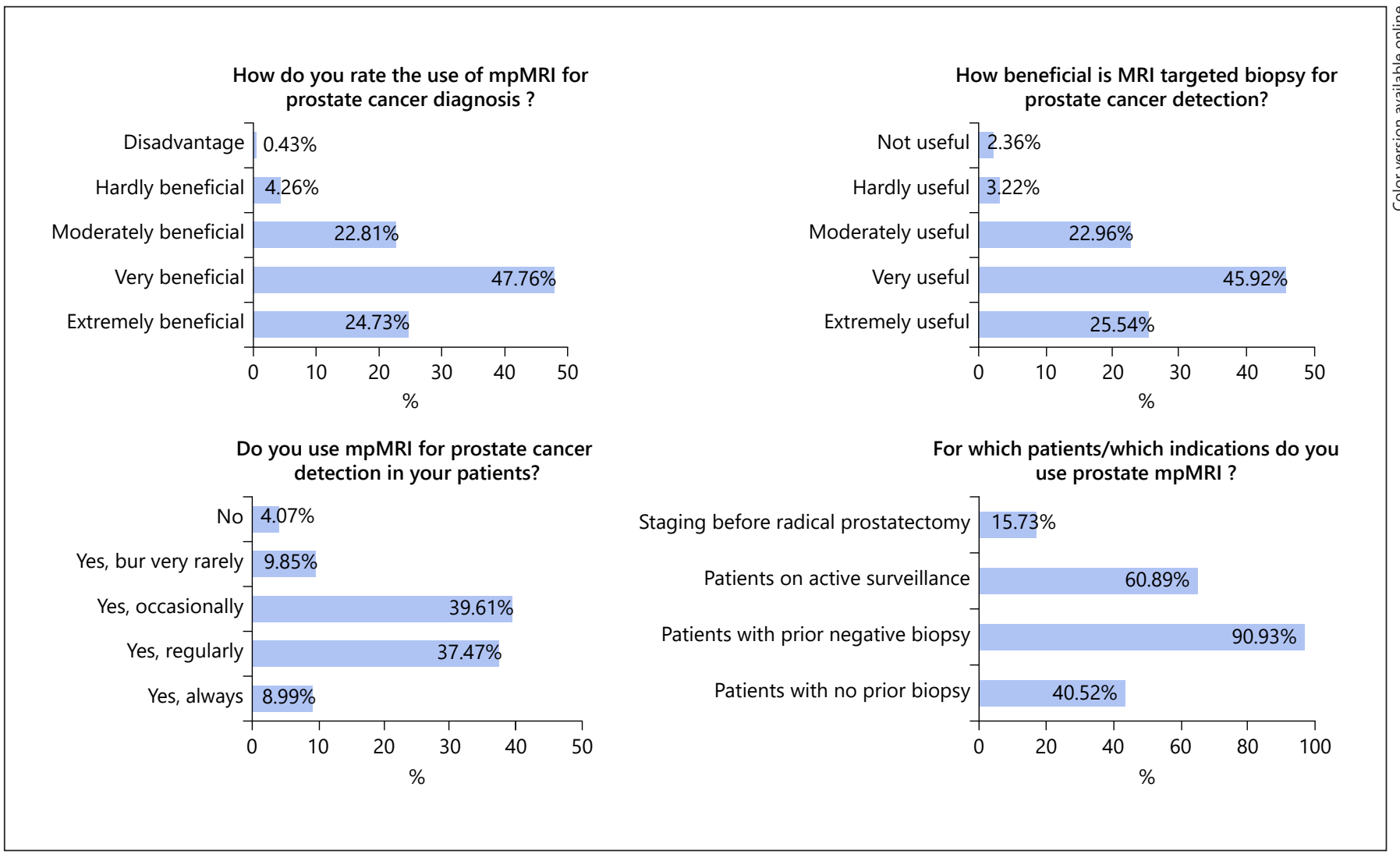

Fig. 2. Selected answers from the participants to the questions of the survey.

Use of Prostate MRI and MRI Targeted Biopsies in Germany 
Table 3. Participants' turnaround time for prostate biopsy and MRI

$\begin{array}{lc}\text { Waiting period for pathological prostate biopsy results }(n=466) \\ 1-3 \text { days } & 229(49.14 \%) \\ 1 \text { week } & 180(38.63 \%) \\ 1-2 \text { weeks } & 55(11.80 \%) \\ >2 \text { weeks } & 2(0.43 \%) \\ \text { Waiting period for a prostate MRI appointment }(n=455) \\ <1 \text { week } & 16(3.52 \%) \\ 1-2 \text { weeks } & 88(19.34 \%) \\ 2-4 \text { weeks } & 219(48.13 \%) \\ >4 \text { weeks } & 116(25.49 \%) \\ \text { Do not use MRI } & 16(3.52 \%)\end{array}$

biopsy core number $>12$ cores (OR 2.219 [1.411; 3.488]; $p=0.001)$ were significantly associated with frequent utilization of prostate mpMRI for PCa diagnosis (Table 4). While an urban setting with $>100,000$ citizens was significant in univariate analysis (OR 1.561 [1.079; 2.258]; $p=0.018$ ), it was not found to be an independent predictor on multivariate analysis $(1.388[0.949 ; 2.029] ; p=$ 0.091 ). Furthermore, practice in a clinic (OR 1.820 [1.138; $2.912] ; p=0.012$ ) was independently associated with considering MRtb useful in practice (Table 4), and the recommendation for MRtb significantly emerged from practice in a clinic (OR 1.775 [1.069; 2.948]; $p=0.027$ ). Interestingly, other techniques such as elastography or ANNAcTRUS were mostly not considered as a meaningful supplement to TRUS ultrasound with ANNAcTRUS as the most likely alternative for particular patients. Noteworthy, most participants waited $<1$ week for pathological results (87.8\%; Table 3).

\section{Discussion}

There is a large discrepancy between the incidence and mortality of PCa [5]. Prostate biopsy has always aimed to detect clinically significant PCa, while "clinically significant" has been defined as a cancer that is likely to progress and that will therefore affect the life expectancy of a particular patient. For decades, systematic ultrasound-guided transrectal prostate biopsy has been the gold standard for a further diagnostic workup of patients suspected to have PCa based on a positive digital rectal exam or an elevated prostate-specific antigen. However, systematic TRUS-guided biopsies suffer from overdiagnosis and overtreatment on one hand, but also from underdiagnosis and undertreatment owing to the poor visibility of $\mathrm{PCa}$ in b-mode ultrasound images as well as the poor accessibility of certain prostate areas (e.g., anterior) through the transrectal route on the other hand. At this point, mpMRI with its detailed morphological and functional information has changed our clinical algorithm. Since 2012, the PI-RADS standard of imaging and reporting prostate MRIs was introduced and improved the detection of clinically significant PCa with a further update in 2019 [6]. Of note, the majority of participants in our survey $(84.4 \%)$ already use the PI-RADS v2 classification to report prostate MRIs, which is in line with another recent survey among urologists and radiologists [7].

Our survey was designed to assess the use of mpMRI and MRtb and more specifically their implementation in urologists' daily practice for the diagnosis and staging of $\mathrm{PCa}$ in Germany. Not surprisingly, mpMRI was proved to be a valuable, widely used tool as $95.9 \%$ of the participants use mpMRI of the prostate at some point and $83.2 \%$ also recommend MRtb. There have been only few surveys, predominantly in the US, indicating a similarly high number of urologists to utilize prostate mpMRI imaging in their daily practice $(85.7-86 \%)$ but a lower number of urologists considering MRtb (60-63\%) [8, 9]. Our study might report a higher acceptance for and application of MRtb since this technique needs some sophisticated equipment and infrastructure and therefore its broader application takes time. A 2013 national survey showed a use of only $34 \%$ for cognitive or ultrasound fusion guided biopsies indicating a strong trend in favor of this technique over time.

The widespread use of mpMRI and MRtb in the diagnosis and management of PCa is also supported by its 60.9 and $54.6 \%$ use in AS patients in our survey. While evidence has mostly been generated retrospectively, an initial analysis concluded that stable findings on mpMRI were predictive of stable Gleason score resulting in fewer biopsies for patients on AS [10]. Furthermore, mpMRI at the time of AS enrollment reduces the misclassification rate of csPCa, and therefore MRtb should be performed in patients with PI-RADS $\geq 3$ [11]. Consequently, the German $S 3$ guideline recommends the use of mpMRI for AS patients [12], and therefrom, application of mpMRI might further increase in Germany for these particular patients. Adding to this, our study again confirms an increasing use of mpMRI with MRtb to improve urologists' confidence recommending AS [13].

Interestingly, several environmental factors show a significant impact on use of mpMRI for PCa diagnosis. Not only the majority of participants lives in cities with $>100,000$ citizens $(54.4 \%)$, but such a setting is also pre- 
Table 4. Uni- and multivariate logistic regression analysis

\begin{tabular}{|c|c|c|c|c|}
\hline \multirow[t]{2}{*}{ Independent characteristics } & \multicolumn{2}{|c|}{ Univariate regression analysis } & \multicolumn{2}{|c|}{ Multivariate regression analysis } \\
\hline & OR [upper; lower KI] & $p$ value & OR [upper; lower KI] & $p$ value \\
\hline \multicolumn{5}{|c|}{ Use of $m p M R I$ for prostate cancer diagnosis (frequently or always versus occasionally or never) } \\
\hline Age ( $\geq 50$ vs. $<50$ years $)$ & $1.158[0.804 ; 1.667]$ & 0.431 & & \\
\hline Gender (F vs. M) & $0.881[0.543 ; 1.432]$ & 0.610 & & \\
\hline Region (West vs. East) & $0.910[0.515 ; 1.606]$ & 0.744 & & \\
\hline Setting ( $\geq 100,000$ vs. $<100,000$ citizens) ${ }^{\mathrm{a}}$ & $1.561[1.079 ; 2.258]$ & 0.018 & $1.388[0.949 ; 2.029]$ & 0.091 \\
\hline Practice type (clinic vs. medical practice) ${ }^{\mathrm{b}}$ & $1.932[1.335 ; 2.797]$ & $<0.001$ & $1.852[1.221 ; 2.808]$ & 0.004 \\
\hline Trained urologist (yes vs. no) & $1.014[0.533 ; 1.928]$ & 0.967 & & \\
\hline Experience ( $>20$ vs. $\leq 20$ years) & $1.258[0.873 ; 1.815]$ & 0.218 & & \\
\hline Self-biopsy (yes vs. no) & $1.032[0.452 ; 2.353]$ & 0.941 & & \\
\hline Core numbers per patient $(>12 \text { vs. } \leq 12)^{\mathrm{c}}$ & $2.468[1.594 ; 3.821]$ & $<0.001$ & $2.219[1.411 ; 3.488]$ & 0.001 \\
\hline \multicolumn{5}{|c|}{ How beneficial is MR targeted biopsy for the diagnosis of prostate cancer (extremely - very useful vs. not-moderately useful) } \\
\hline Age $(\geq 50 \text { vs. }<50 \text { years })^{\mathrm{d}^{\circ}}$ & $0.592[0.386 ; 0.909]$ & 0.017 & $0.587[0.278 ; 1.240]$ & 0.163 \\
\hline Gender (F vs. M) & $1.729[0.928 ; 3.222]$ & 0.084 & & \\
\hline Region (West vs. East) & $1.192[0.627 ; 2.264]$ & 0.592 & & \\
\hline Setting ( $\geq 100,000$ vs. $<100,000$ citizens) & $1.363[0.894 ; 2.081]$ & 0.150 & & \\
\hline Practice type (clinic vs. medical practice) ${ }^{\mathrm{e}}$ & $2.168[1.393 ; 3.375]$ & 0.001 & $1.820[1.138 ; 2.912]$ & 0.012 \\
\hline Trained urologist (yes vs. no) ${ }^{\mathrm{f}}$ & $0.298[0.104 ; 0.859]$ & 0.025 & $0.633[0.196 ; 2.046]$ & 0.445 \\
\hline Experience ( $>20$ vs. $\leq 20$ years) & $0.695[0.455 ; 1.060]$ & 0.091 & & \\
\hline Self-biopsy (yes vs. no) & $1.005[0.386 ; 2.612]$ & 0.992 & & \\
\hline Core numbers per patient $(>12 \text { vs. } \leq 12)^{\mathrm{c}}$ & $1.559[0.930 ; 2.613]$ & 0.092 & & \\
\hline \multicolumn{5}{|c|}{ Would you recommend MR targeted biopsy for prostate cancer diagnosis? (yes vs. no) } \\
\hline Age ( $\geq 50$ vs. $<50$ years) & $0.646[0.394 ; 1.060]$ & 0.084 & & \\
\hline Gender (F vs. M) & $2.019[0.930 ; 4.383]$ & 0.076 & & \\
\hline Region (West vs. East) & $1.136[0.545 ; 2.376]$ & 0.733 & & \\
\hline Setting ( $\geq 100,000$ vs. $<100,000$ citizens) & $1.207[0.739 ; 1.969]$ & 0.452 & & \\
\hline Practice type (clinic vs. medical practice) $)^{g}$ & $1.775[1.069 ; 2.948]$ & 0.027 & $1.775[1.069 ; 2.948]$ & 0.027 \\
\hline Trained urologist (yes vs. no) & $0.642[0.244 ; 1.690]$ & 0.370 & & \\
\hline Experience ( $>20$ vs. $\leq 20$ years) & $0.759[0.466 ; 1.236]$ & 0.268 & & \\
\hline Self-biopsy (yes vs. no) & $0.457[0.105 ; 1.992]$ & 0.297 & & \\
\hline Core numbers per patient ( $>12$ vs. $\leq 12$ ) & $1.490[0.811 ; 2.737]$ & 0.198 & & \\
\hline
\end{tabular}

${ }^{a}$ Multivariate model adjusted for practice type. ${ }^{\mathrm{b}}$ Multivariate model adjusted for age and core numbers. ${ }^{\mathrm{c}}$ Multivariate model adjusted for practice type. ${ }^{\mathrm{d}}$ Multivariate model adjusted for practice type, trained urologist, and experience. ${ }^{\mathrm{e}}$ Multivariate model adjusted for age and trained urologist. ${ }^{\mathrm{f}}$ Multivariate model adjusted for age, gender, practice type, and experience. ${ }^{\mathrm{g}}$ No confounders to be adjusted for.

dictive for an increased use of prostate mpMRI in a univariate analysis $(p=0.018)$. This may be due to a greater availability and higher technical standard in urban agglomerations and might also reflect the technical interest of respondents from such areas. Appropriately, urologists from clinical centers demonstrate a greater use of mpMRI and MRtb compared to urologists from medical practices $(p=0.004)$. These results suggest that short access routes to an mpMRI and greater resources influence decision making in practice since only $36.9 \%$ of those in medical practice routinely perform mpMRI/MRtb compared with $52.2 \%$ in clinical centers and $67.8 \%$ at a university hospi- tal. Furthermore, those in academic centers tend to perform more biopsies and radical prostatectomies and therefore more often utilize MRtb in their practice [8].

Environmental and institutional factors are also reflected as the most common barriers for implementation of MRtb, namely, costs (77.7\%) and linkage with radiology (18.1\%). Interestingly, costs are a nearly as large constraint in the clinic (44.7\%) as in urology practice (58.2\%) probably reflecting the insufficient cost-recovery for mpMRI in daily practice by public as well as private health insurances. Additionally, users have to overcome the higher upfront costs to purchase a fusion prostate biopsy 
device or implement advanced imaging in their daily practice, which additionally requires availability of an expert radiologist or the confidence in reading of prostate mpMRI in community offices. Certainly, such constraints still affect attitudes toward the implementation and use of this technology.

While mpMRI/MRtb is seen as supportive and helpful for the diagnosis of PCa and attitudes toward its use are generally positive, there are some reservations about a universal use for all patients. The majority of respondents believe that mpMRI should be reserved for selected men such as those with a prior negative biopsy (90.9\%) or those under AS (60.9\%). This recommendation is advised in many guidelines $[12,14]$ and reflects the proven effectiveness of prostate mpMRI and MRtb in these clinical situations $[2,15]$. Therefrom, our data suggest that urologists support mpMRI and MRtb for complex patients.

However, the question arises if mpMRI should be performed for all men prior to biopsy to reduce unnecessary interventions, to reduce overdiagnosis of clinically insignificant PCa, and to improve the detection of clinically significant PCa. Only $40.5 \%$ of our respondents would recommend a prostate mpMRI for patients with no prior biopsy, but this is already a large matter of debate. The PROMIS trial included $>500$ biopsy-naïve men and was able to show an avoidance of primary biopsy in $27 \%$ of the patients with the use of mpMRI when used as a triage test before first biopsy [16]. Furthermore, the PRECISION trial reported that prostate mpMRI prior to first biopsy was superior to standard transrectal ultrasound-guided prostate biopsy in men at clinical risk of PCa [4]. Additionally, the question if such an mpMRI first strategy is cost effective has been answered in the PROMIS trial, in which this workflow was shown to be clinically effective and cost effective for the diagnosis of significant PCa [17]. In a position paper on population-based PCa screening, the European Association of Urology (EAU) recommends mpMRI as a test to select biopsy candidates in men at risk of significant $\mathrm{PCa}$ according to prostate-specific antigen levels [18]. However, there is at the moment only a weak recommendation in the EAU guidelines to perform mpMRI before first prostate biopsy [14]. The negative predictive value of mpMRI for PCa remains an important factor and is rated - depending on the patient selection - between 63 and 98\% [19-21]. Overall, mpMRI as a triage test could detect more significant PCa in $40 \%$ of men after prior negative biopsy and in 5\% of biopsynaïve men compared to TRUS-guided biopsy only. Nevertheless, there is a strong recommendation to combine targeted and systematic biopsies as soon as an mpMRI is positive with a PI-RADS $\geq 3$ lesion.

Even though systematic biopsies detect more insignificant and less significant cancers and targeted biopsies detect more significant and less insignificant $\mathrm{PCa}[2,22]$, the combination of targeted and systematic biopsies detects more clinically significant PCa than either modality alone irrespective of a first line approach or in patients with previously negative biopsy [23-25]. The majority of our respondents kept that data in mind and consequently indicated that MRtb does not eliminate the need for systematic biopsies (91\%). However, concerning lesion-targeted biopsies, there seems to be a clear limit for the number of cores per lesion. Most respondents would take a maximum of 4 biopsies per lesion (94.9\%), which is in line with the average number of biopsies per lesion as well as the recommended number of biopsies in larger studies $[4,26]$.

As expected, MRI-ultrasound fusion biopsy is used from the majority of respondents (75.8\%), while cognitive fusion is still appropriate for $26 \%$ and in bore MRI biopsies are rarely used (4.5\%). While all these 3 techniques enhance detection rates for clinically significant PCas compared to standard TRUS-guided biopsies [27], none of these is clearly superior to the others in their detection rate.

There are several limitations in this study. First, the survey design that addressed the members of the German association of urology via email has to be mentioned. This has some reporting bias with a lack of information on candidates that did not respond. A majority of respondents might be more interested in mpMRI and new techniques, therefore being appealed by the technological topic of the survey and its electronic design. Therefrom, we cannot totally generalize the results to the overall urologic community and all members of the German Society of Urology. Also, prostate MRI remains an evolving field, with frequent publication of new trials. Our survey asked urologists' opinions at a single point in time and results could be subject to change with newer results and expert opinions to be published. Finally, this survey represents an assessment of urologists' preferences not necessarily including other disciplines.

\section{Conclusion}

Our data are the first to show the meanwhile high acceptance and clinical use of the prostate mpMRI and MRtb in Germany. Urologists regard mpMRI and MRtb as improving risk stratification for localized $\mathrm{PCa}$ and increasing confidence in using AS. Clinic centers report a 
greater utilization of mpMRI and MRtb than medical practice. However, mpMRI is still not available for all urologists with an excellent image quality and a dedication and experience of radiologic centers. Hence, close collaboration is desirable and will ensure direct interdisciplinary communication between radiologists and urologists while modified mpMRI protocols might be necessary to further reduce costs and increase availability.

\section{Acknowledgements}

We thank the DPKK and the DGU for their kind support with the patient survey. We thank Jokob Schöpe (Department of Medical Biometry, Epidemiology and Informatics, Saarland University, Homburg/Saar, Germany) for his assistance with all statistical calculations.

\section{Statement of Ethics}

All data were collected according to the General Data Protection regulation of the EU. This study was conducted in accordance with the World Medical Association Declaration of Helsinki. The survey does not involve studies of human or animal subjects. Analyzed data were completely anonymized with rigorous data protection measures.

\section{Conflict of Interest Statement}

The authors declare that they have no conflicts of interests to declare.

\section{Funding Sources}

No funding was received for this study.

\section{Author Contributions}

M.S.: project development, data collection, data analysis and interpretation, manuscript writing, and critical revision. J.L.: project development, data collection, data analysis and interpretation, and critical revision. G.K. and B.W: project development, data analysis and interpretation, manuscript writing, critical revision, and supervision. A.B., S.F., K.U., and L.B.: project development, data interpretation, language revision, and critical revision.

\section{References}

1 Stabile A, Giganti F, Rosenkrantz AB, Taneja SS, Villeirs G, Gill IS, et al. Multiparametric MRI for prostate cancer diagnosis: current status and future directions. Nat Rev Urol. 2020 Jan; 17(1):41-61.

2 Siddiqui MM, Rais-Bahrami S, Turkbey B, George AK, Rothwax J, Shakir N, et al. Comparison of MR/ultrasound fusion-guided biopsy with ultrasound-guided biopsy for the diagnosis of prostate cancer. JAMA. 2015 Jan 27;313(4):390-7.

3 Klotz L, Pond G, Loblaw A, Sugar L, Moussa M, Berman D, et al. Randomized study of systematic biopsy versus magnetic resonance imaging and targeted and systematic biopsy in men on active surveillance (ASIST): 2-year postbiopsy follow-up. Eur Urol. 2020 Mar;77(3):311-7.

4 Kasivisvanathan V, Rannikko AS, Borghi M, Panebianco V, Mynderse LA, Vaarala MH, et al. MRI-targeted or standard biopsy for prostate-cancer diagnosis. N Engl J Med. 2018 May 10;378(19):1767-77.

5 Siegel RL, Fedewa SA, Miller KD, GodingSauer A, Pinheiro PS, Martinez-Tyson D, et al. Cancer statistics for Hispanics/Latinos, 2015. CA Cancer J Clin. 2015;65(6):457-80.

6 Turkbey B, Rosenkrantz AB, Haider MA, Padhani AR, Villeirs G, Macura KJ, et al. Prostate Imaging Reporting and Data System Version 2.1: 2019 update of prostate imaging reporting and data system version 2. Eur Urol. 2019 Sep;76(3):340-51.
7 Spilseth B, Ghai S, Patel NU, Taneja SS, Margolis DJ, Rosenkrantz AB. A comparison of radiologists' and urologists' opinions regarding prostate MRI reporting: results from a survey of specialty societies. AJR Am J Roentgenol. 2018 Jan;210(1):101-7.

8 Muthigi A, Sidana A, George AK, Kongnyuy M, Maruf M, Valayil S, et al. Current beliefs and practice patterns among urologists regarding prostate magnetic resonance imaging and magnetic resonance-targeted biopsy. Urol Oncol. 2017 Jan;35(1):32.e1-7.

9 Tooker GM, Truong H, Pinto PA, Siddiqui MM. National survey of patterns employing targeted MRI/US guided prostate biopsy in the diagnosis and staging of prostate cancer. Curr Urol. 2019 Mar 8;12(2):97-103.

10 Walton Diaz A, Shakir NA, George AK, RaisBahrami S, Turkbey B, Rothwax JT, et al. Use of serial multiparametric magnetic resonance imaging in the management of patients with prostate cancer on active surveillance. Urol Oncol. 2015 May;33(5):202.e1-7.

11 Luzzago S, Musi G, Catellani M, Russo A, Di Trapani E, Mistretta FA, et al. Multiparametric magnetic-resonance to confirm eligibility to an active surveillance program for low-risk prostate cancer: intermediate time results of a third referral high volume centre active surveillance protocol. Urol Int. 2018;101(1):5664.
12 Leitlinienprogramm Onkologie (Deutsche Krebsgesellschaft, Deutsche Krebshilfe, AWMF) Langversion (2019) Interdisziplinäre Leitlinie der Qualität S3 zur Früherkennung, Diagnose und Therapie der verschiedenen Stadien des Prostatakarzinoms, 5.1, AWMF Registernummer: 043/022OL [cited 2019 Dec 17]. Available from: http://www.leitlinienprogramm-onkolo-gie.de/leitlinien/prostatakarzinom/.

13 Bukavina L, Tilburt JC, Konety B, Shah ND Gross CP, Yu JB, et al. Perceptions of prostate MRI and fusion biopsy of radiation oncologists and urologists for patients diagnosed with prostate cancer: results from a national survey. Eur Urol Focus. 2020 Mar 15;6(2): 273-9.

14 EAU Guidelines. Edn. Presented at the EAU Annual Congress Barcelona 2019.

15 Guo R, Cai L, Fan Y, Jin J, Zhou L, Zhang K. Magnetic resonance imaging on disease reclassification among active surveillance candidates with low-risk prostate cancer: a diagnostic meta-analysis. Prostate Cancer Prostatic Dis. 2015 Sep;18(3):221-8.

16 Ahmed HU, El-Shater Bosaily A, Brown LC, Gabe R, Kaplan R, Parmar MK, et al. Diagnostic accuracy of multi-parametric MRI and TRUS biopsy in prostate cancer (PROMIS): a paired validating confirmatory study. Lancet. 2017 Feb 25;389(10071):815-22. 
17 Faria R, Soares MO, Spackman E, Ahmed HU, Brown LC, Kaplan R, et al. Optimising the diagnosis of prostate cancer in the era of multiparametric magnetic resonance imaging: a cost-effectiveness analysis based on the prostate MR imaging study (PROMIS). Eur Urol. 2018 Jan;73(1):23-30.

18 Gandaglia G, Albers P, Abrahamsson PA, Briganti A, Catto JWF, Chapple CR, et al. Structured population-based prostate-specific antigen screening for prostate cancer: the European Association of Urology Position in 2019. Eur Urol. 2019 Aug;76(2):142-50.

19 Abd-Alazeez M, Ahmed HU, Arya M, Charman SC, Anastasiadis E, Freeman A, et al The accuracy of multiparametric MRI in men with negative biopsy and elevated PSA level: can it rule out clinically significant prostate cancer? Urol Oncol. 2014 Jan;32(1):45.e1722.

20 Fütterer JJ, Briganti A, De Visschere P, Emberton M, Giannarini G, Kirkham A, et al Can clinically significant prostate cancer be detected with multiparametric magnetic reso- nance imaging? A systematic review of the literature. Eur Urol. 2015 Dec;68(6):1045-53.

21 Sivaraman A, Sanchez-Salas R, Ahmed HU, Barret E, Cathala N, Mombet A, et al. Clinical utility of transperineal template-guided mapping biopsy of the prostate after negative magnetic resonance imaging-guided transrectal biopsy. Urol Oncol. 2015 Jul;33(7): 32911.e7-11.

22 Meng X, Rosenkrantz AB, Mendhiratta N, Fenstermaker M, Huang R, Wysock JS, et al. Relationship between prebiopsy multiparametric magnetic resonance imaging (MRI), biopsy indication, and MRI-ultrasound fusion-targeted prostate biopsy outcomes. Eur Urol. 2016 Mar;69(3):512-7.

23 Filson CP, Natarajan S, Margolis DJ, Huang J, Lieu P, Dorey FJ, et al. Prostate cancer detection with magnetic resonance-ultrasound fusion biopsy: the role of systematic and targeted biopsies. Cancer. 2016 Mar 15;122(6):884-92.

24 Borkowetz A, Renner T, Platzek I, Toma M, Herout R, Baunacke M, et al. Evaluation of transperineal magnetic resonance imaging/ul- trasound-fusion biopsy compared to transrectal systematic biopsy in the prediction of tumour aggressiveness in patients with previously negative biopsy. Urol Int. 2019;102(1):20-6.

25 Tu X, Liu Z, Zhang C, Chang T, Xu H, Bao Y, et al. Diagnostic role of magnetic resonance imaging-targeted biopsy for prostate cancer in biopsy-naïve men: a meta-analysis. Urol Int. 2020:104(3-4):187-98.

26 Valerio M, Donaldson I, Emberton M, Ehdaie B, Hadaschik BA, Marks LS, et al. Detection of clinically significant prostate cancer using magnetic resonance imaging-ultrasound fusion targeted biopsy: a systematic review. Eur Urol. 2015 Jul;68(1):8-19.

27 Wegelin O, van Melick HHE, Hooft L, Bosch JLHR, Reitsma HB, Barentsz JO, et al. Comparing three different techniques for magnetic resonance imaging-targeted prostate biopsies: a systematic review of in-bore versus magnetic resonance imaging-transrectal ultrasound fusion versus cognitive registration. Is there a preferred technique? Eur Urol. 2017 Apr;71(4):517-31. 